\title{
Tratamento convencional e endovascular para rara associação de doenças: síndrome de veia cava superior e aneurisma aorto-ilíaco - controle após 12 meses
}

\author{
Conventional and endovascular treatment for a rare combination of diseases: superior vena \\ cava syndrome and aortoiliac aneurysm - control after 12 months \\ Gustavo Petorossi Solano', Rodrigo Andrade Vaz de Melo', Luis Claudio Rosa Arantes', Daniel Queiroz Neves², Márcio \\ Cerbazzi Tavares Cardoso 3 , Mauro Henrique de Lima33, Sergio Lopes de Azevedo ${ }^{4}$, Paulo Eduardo Ocke Reis ${ }^{5}$
}

\begin{abstract}
Resumo
A associação entre a síndrome de veia cava superior e uma dilatação aneurismática das artérias aorta e ilíacas não é comum. A abordagem de cada uma destas patologias pode ser efetuada através do modo convencional, com cirurgia aberta ou pela técnica endovascular. Neste trabalho, relatamos as duas modalidades de intervenção cirúrgica executadas e discutimos suas indicações e os resultados deste caso em particular.
\end{abstract}

Palavras-chave: síndrome de veia cava superior; aneurisma ilíaco; prótese vascular.

\begin{abstract}
The association between superior vena cava syndrome and an aorto-iliac aneurysm is not common. The approach to each of theses diseases can be either by the conventional way with open surgery or by endovascular techniques. We report the two methods of surgical intervention and discuss their indications and results in this particular case.

Keywords: superior vena cava syndrome; iliac aneurysm; blood vessel prosthesis.
\end{abstract}

Introdução

A trombose da veia cava superior e de outras veias centrais não é um fato raro, porém as doenças benignas são causas menos comuns, representando cerca de 15 a $22 \%$ dos casos ${ }^{1}$. A trombose venosa central induzida pelo procedimento de cateterismo tem se tornado a principal causa de origem benigna frente ao crescente número de procedimentos, como acessos para hemodiálise e monitoramento invasivo ou implante de marcapasso $^{2-4}$. Entretanto, a associação da síndrome de veia cava superior com um aneurisma aorto-ilíaco torna esse caso ainda mais raro. No presente trabalho, relatamos a abordagem convencional e endovascular para as patologias do paciente, discutimos indicações e a ordem de abordagem e apresentamos os resultados após 12 meses de seguimento.

\section{Relato do caso}

Paciente masculino, de 56 anos, procurou o setor de emergência queixando-se de falta de ar e vermelhidão no rosto. Após episódio de bradicardia e síncope há 11 anos, recebeu implante de um marca-passo definitivo com seu módulo gerador fixado em região infraclavicular direita e há 3 anos, por migração inframamária, foi realizada

Trabalho realizado no Hospital Universitário Antônio Pedro (HUAP) - Universidade Federal Fluminense (UFF) - Niterói (RJ), Brasil.

'Staff do Serviço de Cirurgia Vascular do HUAP-UFF - Niterói (RJ), Brasil.

${ }^{2}$ Residente do Serviço de Cirurgia Vascular do HUAP-UFF - Niterói (RJ), Brasil.

${ }^{3}$ Médico pós-graduando do Serviço de Cirurgia Vascular do HUAP-UFF - Niterói (RJ), Brasil.

${ }^{4}$ Enfermeiro do Serviço de Cirurgia Vascular do HUAP-UFF - Niterói (RJ)), Brasil.

${ }^{5}$ Chefe do Serviço de Cirurgia Vascular do HUAP-UFF - Niterói (RJ), Brasil.

Conflito de interesses: nada a declarar.

Submetido em: 16.07.10. Aceito em: 03.06.11.

J Vasc Bras. 2011;10(3):217-221. 
troca deste gerador em cirurgia ambulatorial, com manipulação dos cabos do marca-passo. Após seis meses deste evento, iniciou-se um quadro de opressão torácica intermitente, dispnéia, edema e pletora facial, sendo referidos aumento progressivo da intensidade e frequência. Ao exame físico observava-se dispnéia em repouso, edema cervical, torácico e de membros superiores, intensa pletora facial e uma massa abdominal pulsátil em hipogástrio com extensão à região inguinal esquerda. Exames laboratoriais se mostraram sem alterações e a radiografia de tórax indicava apenas alargamento do mediastino, atribuído à insuficiência cardíaca congestiva prévia. A ultrassonografia de tireóide foi normal, a coronariografia também não evidenciou lesões e à endoscopia digestiva alta observavam-se varizes esofagianas em terço superior. Realizado ecocardiograma transesofágico que mostrava imagem sugestiva de trombo pericateter do marca-passo. A tomografia computadorizada de tórax não evidenciou adenopatia peri-hilar ou massa mediastinal ou pulmonar, confirmando a origem benigna da patologia. Observava-se a presença de fibrose em veia cava superior, justa-atrial (Figura 1). A tomografia computadorizada (TC) de abdome e pelve mostrou a presença de aneurisma de aorta em terço distal com $3 \mathrm{~cm}$ de diâmetro e aneurisma bilateral de artéria ilíaca, que media $2 \mathrm{~cm}$ à direita e $6 \mathrm{~cm}$ à esquerda (Figura 2). Foi realizada, então, uma flebografia do paciente, em que se evidenciou fluxo lentificado no tronco braquiocefálico esquerdo (TBC), inversão do fluxo da veia ázigos e grave estenose da veia cava superior em sua porção justa-cardíaca, imagens

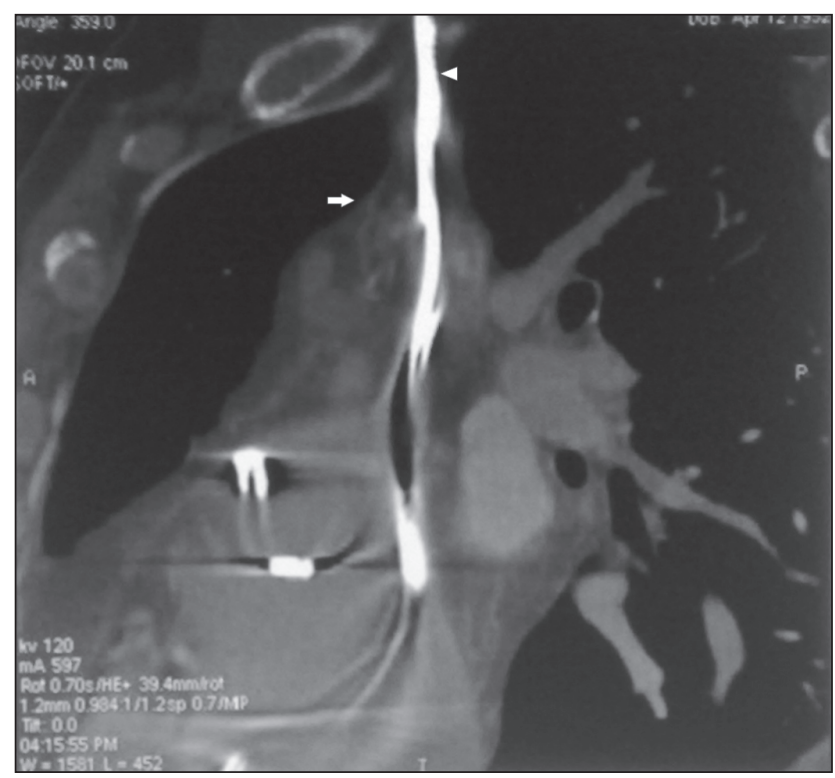

Figura 1. Fibrose de veia cava superior justa-atrial.

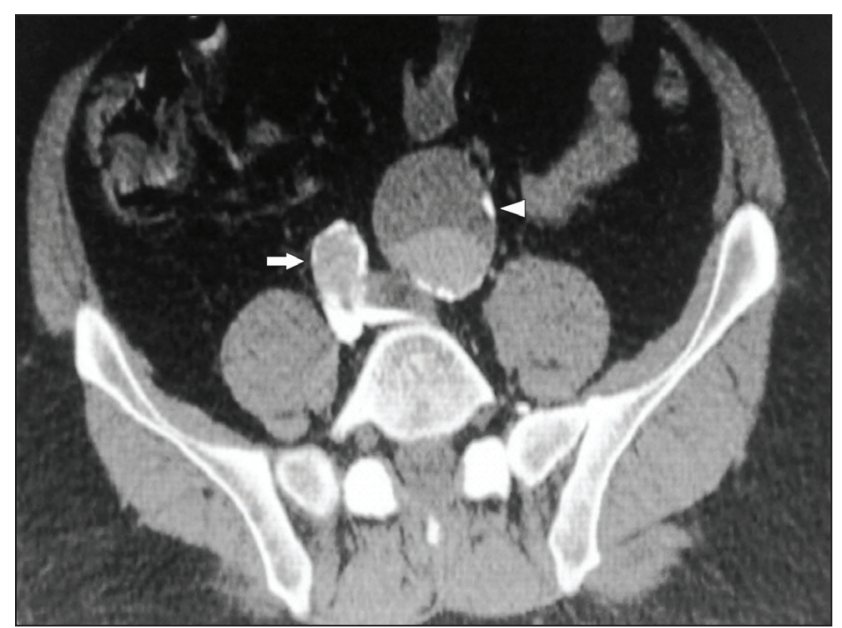

Figura 2. Aneurismas das artérias ilíacas.

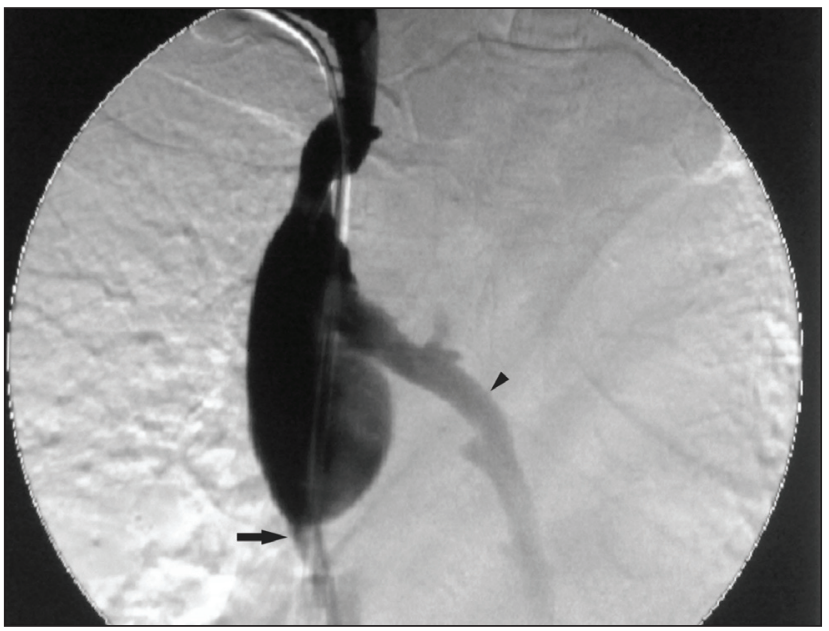

Figura 3. Cavografia - estenose justa-atrial e inversão do fluxo da veia ázigos.

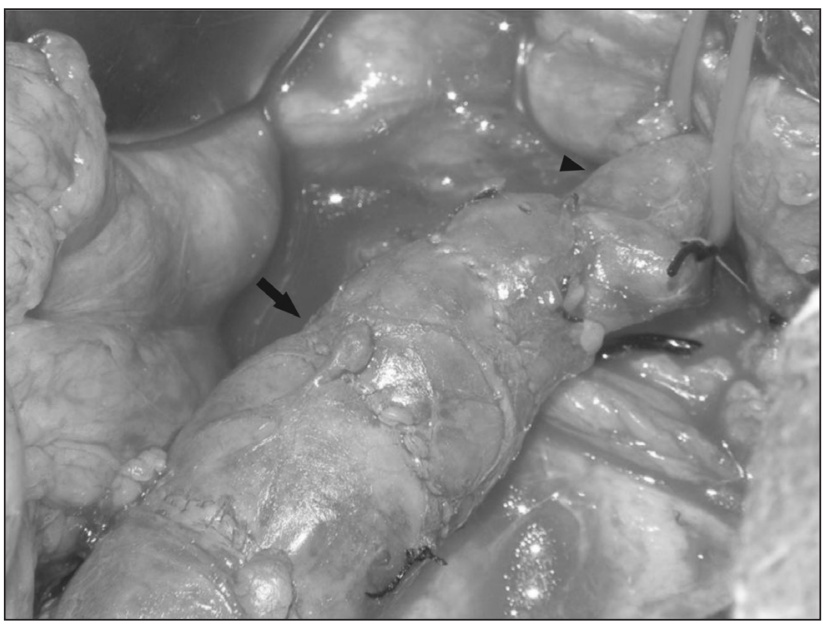

Figura 4. Anastomose do enxerto de veia safena espiralada com o tronco braquiocefálico venoso esquerdo. 


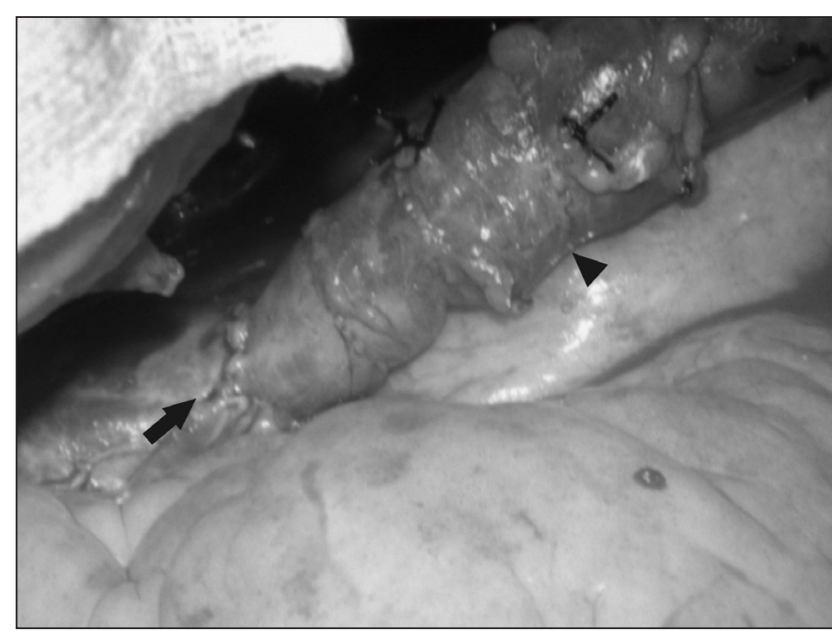

Figura 5. Anastomose do enxerto com o átrio direito.

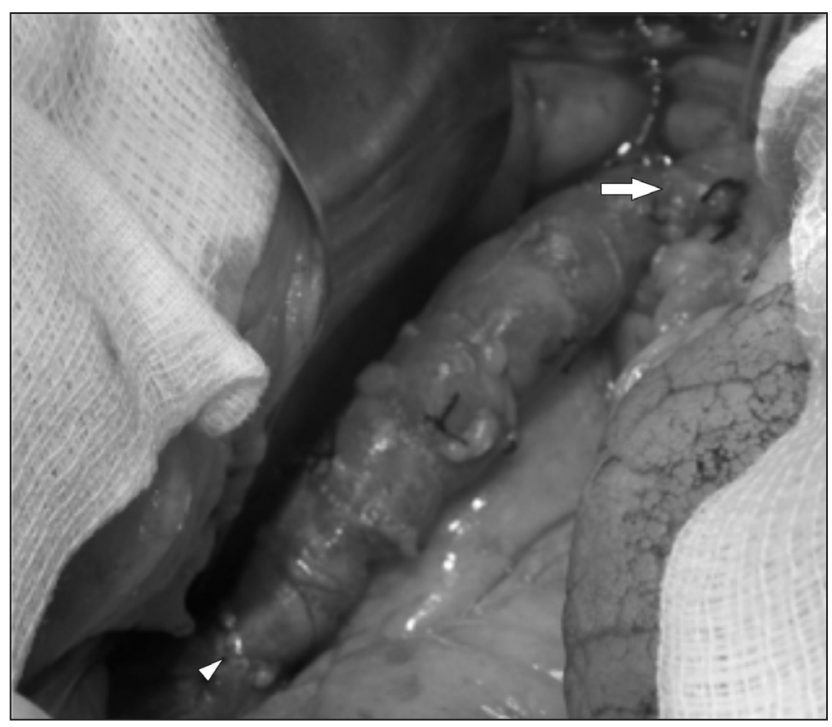

Figura 6. Resultado final após liberação do fluxo pelo enxerto.

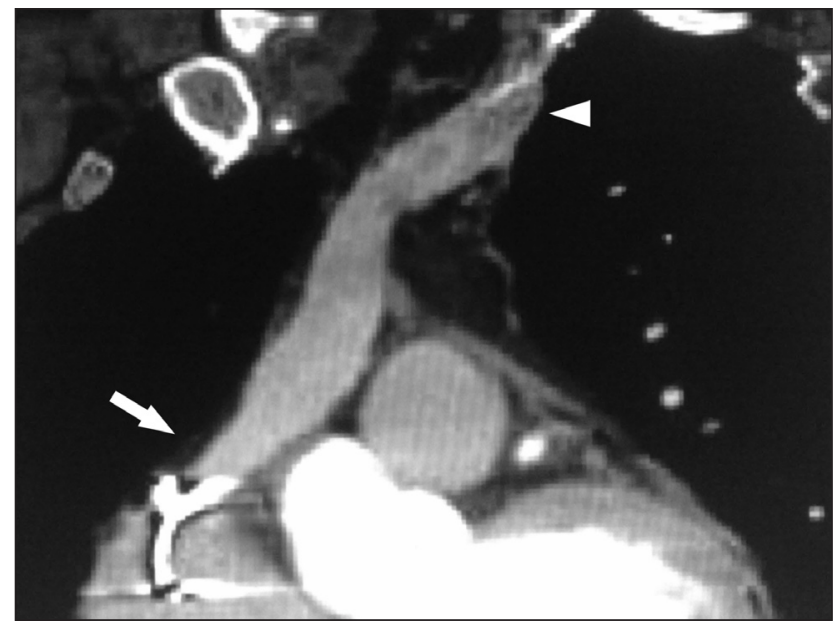

Figura 7. Controle após 12 meses - patência do enxerto. compatíveis com síndrome de veia cava superior tipo III (Figura 3). Após 12 dias de ampla investigação e com o paciente em repouso absoluto no leito, com a cabeceira elevada a $60^{\circ}$, recebendo suporte clínico e nutricional, optou-se inicialmente pela cura do aneurisma aorto-ilíaco com implante de endoprótese Zenith aorto-monoilíaca esquerda com embolização da artéria ilíaca interna esquerda e ponte fêmoro-femoral cruzada com PTFE. O procedimento foi realizado sem intercorrências, com o paciente sob anestesia geral e intubação orotraqueal. $\mathrm{Na}$ evolução pós-operatória, não houve intercorrência clínica e palpavam-se pulsos amplos bilateralmente em membros inferiores. O paciente recebeu alta hospitalar em dois dias, sob orientações e com o quadro respiratório compensado, sendo programada reinternação em duas semanas. Em um segundo tempo, foi realizada ponte do tronco braquiocefálico venoso esquerdo ao átrio direito, com enxerto de veia safena interna espiralada (Figuras 4, 5 e 6). O paciente evoluiu com deiscência da sutura esternal após sete dias, quando foi levado à nova intervenção cirúrgica para ressutura esternal. Foi mantida anticoagulação oral, com o paciente recebendo alta do CTI após 14 dias, a contar do primeiro procedimento, quando já se observava regressão completa dos sinais e sintomas. Desde a alta hospitalar, o paciente permanece em acompanhamento ambulatorial, com regressão dos sintomas e sem queixas de nenhuma espécie, executando normalmente suas atividades diárias. Realizada angiotomografia de controle após 12 meses, que confirmou a patência do enxerto torácico (Figuras 7) e exclusão do aneurisma, com redução do seu diâmetro para $4,8 \mathrm{~cm}$ em artéria ilíaca comum esquerda (Figura 8) e sem evidências de vazamento (Figura 9).

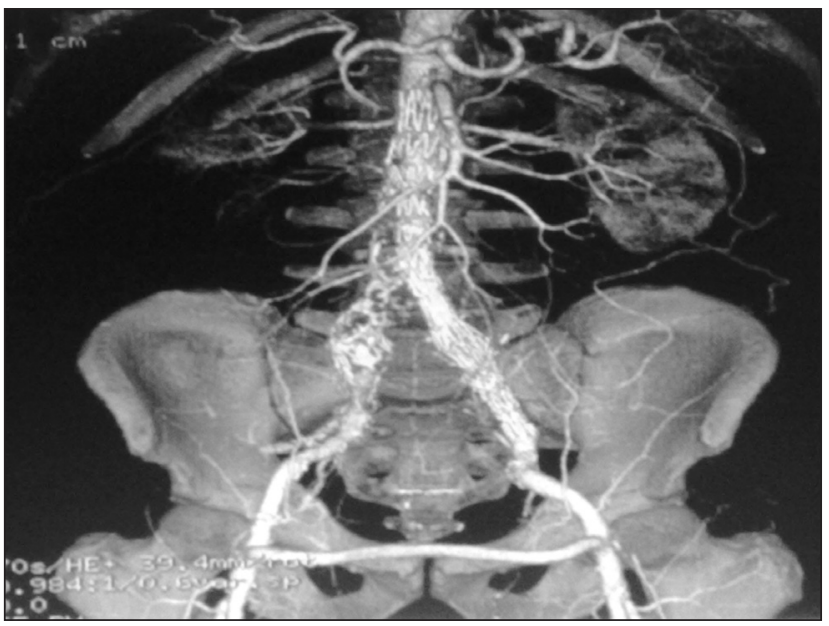

Figura 8. Controle após 12 meses - aneurisma aorto-ilíaco excluído. 


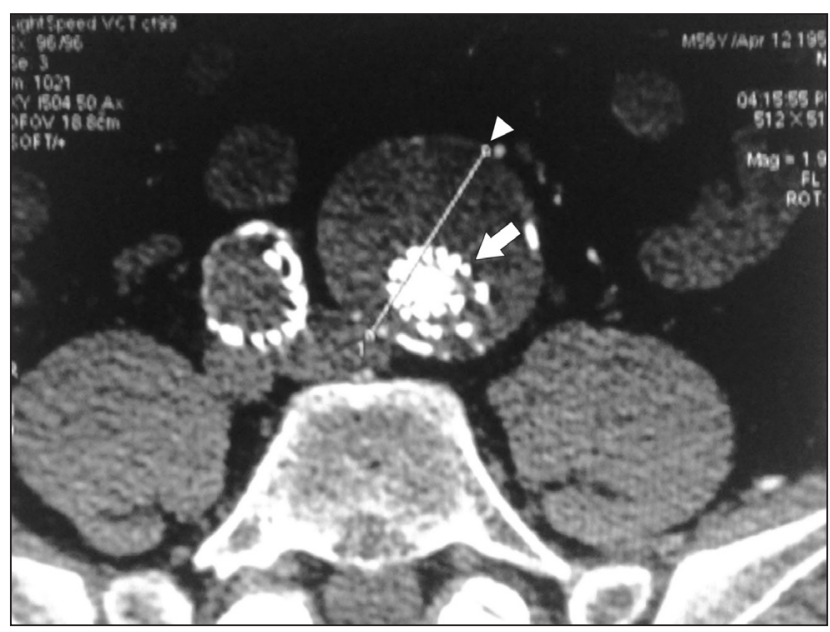

Figura 9. Controle após 12 meses - redução do diâmetro do aneurisma em artéria ilíaca comum esquerda.

\section{Discussão}

A síndrome de veia cava superior de origem benigna é uma patologia rara, porém com prevalência crescente em virtude do aumento de procedimentos endovasculares. Conforme apresentado, a presença de aneurisma aorto-ilíaco associado torna o caso ainda mais peculiar. Considerando o tratamento, ambas as patologias podem ser abordadas da forma cirúrgica convencional ou utilizando-se a técnica endovascular. Nos casos de doença maligna, a recanalização endovascular deve claramente ser o tratamento de escolha $\mathrm{a}^{5-9}$, com a reconstrução cirúrgica sendo reservada para os casos de tumores ressecáveis ${ }^{10-13}$. No caso da síndrome de veia cava superior de origem benigna, a técnica cirúrgica convencional permanece atualmente como primeira opção de tratamento ${ }^{14-17}$, embora existam autores que mantenham a indicação do tratamento endovascular, apesar do custo de múltiplas intervenções secundárias e da necessidade de um maior tempo de seguimento dos $\operatorname{casos}^{18-20}$. Este caso trata de um paciente jovem, com extensa trombose venosa crônica (tipo III) e não susceptível à reconstrução endovascular em virtude da localização da lesão (justa-atrial). Além desses casos, a cirurgia aberta é ainda indicada naqueles pacientes com lesões menos extensas (tipo I ou II) que não foram beneficiados pela terapia endovascular ${ }^{1}$.

Por outro lado, considerando-se a abordagem do aneurisma aorto-ilíaco, entendemos que intervir neste paciente através do método convencional aumentaria demasiadamente o risco cirúrgico. A ordem de abordagem dessas patologias é outro fator importante a ser avaliado. Sabemos que em pacientes com dilatações aneurismáticas da aorta, é grande a chance de rotura no decorrer de outro procedimento cirúrgico ou mesmo no pós-operatório. Analisando estes dados, optamos por tratar inicialmente o aneurisma aorto-ilíaco. A técnica endovascular foi escolhida, neste caso, visando um menor trauma cirúrgico e uma recuperação mais rápida do paciente, que seria submetido à outra intervenção em seguida. Ambos os procedimentos foram bem sucedidos e, após 12 meses, o paciente permanece com regressão completa dos sinais e sintomas, em acompanhamento ambulatorial.

\section{Conclusão}

Com base no que foi apresentado, concluímos que as técnicas de abordagem cirúrgica para a síndrome de veia cava superior de origem benigna e doença aneurismática aorto-ilíaca devem ser amplamente discutidas a fim de executar-se o melhor para o paciente. A concomitância de lesões obriga-nos ainda a decidir qual a melhor sequência de intervenção. Por se tratar de um caso raro, é necessário ainda um maior tempo de seguimento, porém a associação da técnica endovascular e convencional na abordagem de determinadas patologias pode trazer grandes benefícios ao paciente.

\section{Agradecimentos}

Agradecemos às Dras. Helen Christian Pessoni, Lucia Helena Soares e Maria Eduarda Tavares, e aos Drs. Celso Luis Chouin, Wellinton Draxler Pereira, Victor Luís Correa e Dr. Tiago Mafort pela contribuição na condução deste caso.

\section{Referências}

1. Oderich GS, Gloviczki P. Síndrome de veia cava superior. In: Brito C). Cirurgia Vascular, Cirurgia Endovascular, Angiologia. 2nd ed. Rio de Janeiro: Revinter; 2008. p. 1618-31.

2. Doty DB, Doty JR, Jones KW. Bypass of superior vena cava. Fifteen years' experience with spiral vein graft for obstruction of superior vena cava caused by benign disease. J Thorac Cardiov Surg. 2000; 99(5):889-95.

3. Gloviczki P, Pairolero PC, Toomey BJ, et al. Reconstruction of large veins for nonmalignant venous occlusive disease. J Vasc Surg. 1992; 6(5):750-61.

4. Lin $\mathrm{CT}$, Kuo CT, Lin KH, et al. Superior vena cava syndrome as a complication of transvenous permanent pacemaker implantation. Jpn Heart J. 1999;40(4):477-80.

5. Kastner RJ, Fisher WG, Blacky AR, et al. Pacemaker-induced superior vena cava syndrome with successful treatment by ballon venoplaty. Am J Cardiol. 1996;77(9):789-90. 
6. Porte $H$, Metois $D$, Finzi $L$ et al. Superior vena cava syndrome of malignant origin. Which surgical procedure for which diagnosis? Eur J Cardiothorac Surg. 2000;17(4):384-8.

7. Da Inês D, Chabrot P, Cassagnes L, Merle et al. O tratamento endovascular da síndrome do SVC de origem neoplásica: uma revisão de 34 casos. J Radiol. 2008;89(7-8 Pt 1):881-90.

8. Lanciego C, Pangua C, Chacón Jl, et al. Endovascular stenting as the first step in the overall management of malignant superior vena cava syndrome. AJR Am J Roentgenol. 2009;193(2):549-58.

9. Nguyen NP, Borok TL, Welsh J, et al. Safety and effectiveness of vascular endoprosthesis for malignant superior vena cava syndrome. Thorax. 2009; 64(2):174-8.

10. Moore W, Hollier LH. Reconstruction of the superior vena cava and central veins. In Bergan J, Yao JST. (editores). Venous disorders. Philadelphia: W.B. Saunders; 1991. p. 517-27.

11. Dartevelle PG, Chapelier AR, Pastorino U, et al. Long-term followup after prosthetic replacement of the superior vena cava combined with resection of mediastinal-pulmonary malignant tumors. J Thorac Cardiovasc Surg. 1991;102(2):259-65.

12. Magnan PE, Thomas P, Giudicelli R, et al. Surgical reconstruction of the superior vena cava. Cardiovasc Surg. 1994;2(5):598-604.

13. Gloviczki P, Bower TC, McKusick M, Pailorolero PC. Superiorvena cava syndrome; Endovascular and direct surgical treatment. In: Gloviczki P, Yao JST. (editores). Handbook of venous disorders. London: Chapman \& Hall, 1996. p. 580-99.

14. Kalra M, Gloviczki P, Andrews JC, et al. Open surgical and endovascular treatment of superior vena cava syndrome caused by nonmalignant disease. J Vasc Surg. 2003;38(2):215-23.
15. Alimi YS, Gloviczki P, Vrtiska T), et al. Reconstruction of the superior vena cava: benefits of postoperative surveillance and secondary endovascular interventions. J Vasc Surg. 1998;27(2):287-301.

16. Picquet J, Blin V, Dussaussoy $C$, et al. Surgical reconstruction of the superior vena cava system: indications and results. Surgery. 2009; 145(1):93-9.

17. Erbella J, Hess PJ, Huber TS. Superior vena cava bypass with superficial femoral vein for benign superior vena cava syndrome. Ann Vasc Surg. 2006;20(6):834-8.

18. Bornak A, Wicky S, Ris HB, et al. Endovascular treatment of stenoses in the superior vena cava syndrome caused by non-tumoral lesions. Eur Radiol. 2003;13(5):950-6.

19. Rizvi AZ, Kalra M, Biarnason H, Bower TC, Schleck C, Gloviczki P. Benign superior vena cava syndrome: stenting is now the first line of treatment. J Vas Surg 2008;47(2):372-80.

20. Cardozo MA, Lichtenfels E, Erling Junior. N, et al. Tratamento endovascular da síndrome da veia cava superior: relato de caso e revisão da literatura. J Vasc Bras. 2006;5(4):308-12.

Correspondência
Gustavo Petorossi Solano
Rua Doutor Mário Viana 405 - apto 1.006 - Santa Rosa
CEP 24241-000 - Niterói (RJ), Brasil
E-mail: contato@gustavosolano.com.br
Contribuições dos autores
Concepção e desenho do estudo: GPS, PEOR
Análise e interpretação dos dados: GPS, PEOR, LCRA, RAVM
Coleta de dados: GPS, MCTV, DQN, MHL, SLA
Redação do artigo: GPS
Revisão crítica do texto: PEOR, DQN
Aprólise estatística: N/A.
*Todos os autores leram e aprovaram a versão final submetida ao J Vasc Bras.

